\title{
Shigelosis en el Cantón de los Chiles durante el año 2016
}

\author{
Jorge Mauricio Montero García \\ Licenciado en Microbiología y Química Clínica. Microbiólogo Químico Clínico. Hospital de Los Chiles, Sección de \\ Bacteriología, Caja Costarricense del Seguro Social. Máster en Gerencia de Proyectos. mont26@hotmail.com
}

Recibido: 03 julio 2017

Aceptado: 20 octubre 2017

\begin{abstract}
RESUMEN
El género Shigella está constituido por cuatro especies, Shigella sonnei, S. flexneri, S. dysenteriae y S. boydii todas con capacidad patógena. Estas bacterias son las causantes de la disentería bacilar cuya gravedad depende del serogrupo de la bacteria, además, de factores inmunitarios, nutrición y edad del hospedero. Durante el año 2016 en el cantón de Los Chiles hubieron 39 aislamientos confirmados de Shigella spp., siendo $S$. sonnei la más frecuente con el $75 \%$ de los casos y $S$. flexneri con $25 \%$. El grupo etáreo más afectado fue el de los niños menores a 12 años y el distrito con una mayor cantidad de episodios reportados fue el de Los Chiles. El patrón de sensibilidad se comportó de manera similar al reportado a nivel nacional. Se presenta un análisis descriptivo de la información clínico epidemiológica y de laboratorio de los casos confirmados en el Laboratorio Clínico del Hospital de Los Chiles durante el año 2016.
\end{abstract}

Palabras clave: Shigelosis, disentería bacilar, Shigella, antibiótico.

\footnotetext{
ABSTRACT

The genus Shigella consists of four species, Shigella sonnei, S. flexneri, S. dysenteriae and S. boydii, all with pathogenic capacity. These bacteria are the cause of bacillary dysentery whose severity depends on the serogroup of the bacteria, in addition, of immune factors, nutrition and age of the host. During the year 2016 in the canton of Los Chiles there were 39 confirmed isolates of Shigella spp., being S. sonnei the most frequent with $75 \%$ of the cases and $S$. flexneri with $25 \%$. The most affected age group was children under 12 years of age and the district with the highest number of reported episodes was Los Chiles. The sensitivity pattern behaved in a manner similar to that reported at the national level. We present a descriptive analysis of the clinical and epidemiological information of the cases confirmed in the Clinical Laboratory of the Hospital of Los Chiles during the year 2016 .
}

Key words: Shigellosis, bacillary dysentery, Shigella, antibiotic.

\section{Introducción}

El género Shigella pertenece a la familia Enterobacteriaceae y está constituido por cuatro especies; Shigella sonnei, Shigella flexneri, Shigella boydii y Shigella dysenteriae (Lampel \& Maurelli, 2003) todas con capacidad patógena. Esta bacteria es un bacilo Gram negativo, anaerobio facultativo, no fermentador de lactosa, no móvil, no formador de esporas (Public Health England, 2015)

La shigelosis o disentería bacilar se caracteriza por causar enteritis invasora distinguida por producir dolor abdominal cólico, diarrea, fiebre, tenesmo y algunas veces toxemia (Ramírez, 2002). La afectación colónica da lugar a una reacción inflamatoria intensa con moco y pus, pudiendo formarse úlceras sangrantes, por lo que las deposiciones son, característicamente, de pequeño volumen y pueden ir acompañadas de moco y sangre dando lugar, en conjunto, al cuadro denominado disentería bacilar (Prats y Mirelis, n.d), sin embargo en muchos casos se presenta la diarrea acuosa como cuadro inicial (Ramírez, 2002).

La gravedad de la infección y la tasa de mortalidad dependen de factores como la edad, el estado nutricional e inmunológico de la persona afectada y el serogrupo y serotipo de la bacteria, siendo Shigella dysenteriae la más patógena (Baca et al, 2013). En shigelosis el uso de antibióticos se utiliza terapéuticamente para disminuir la duración del cuadro clínico, acortar el 
período de transmisión del agente causal y evitar las complicaciones (Del Piano, Tejerina, Conat y Aviles, 2001). La creciente resistencia a antimicrobianos, limita el uso de tratamiento empírico (Bernardes et al., 2013).

La enteritis por Shigella spp. sigue siendo una causa importante de cuadros de diarrea a nivel mundial, causando anualmente un millón de muertes en países en vías de desarrollo (Keddy et al., 2012, Mota et al. 2010) por lo que es de suma importancia un adecuado control y vigilancia epidemiológica. Según el decreto ejecutivo 37.306-S de Reglamento de Vigilancia de la Salud, en nuestro país, los casos de shigelosis son de reporte obligatorio por medio de las boletas VE-01 con las cuales se da alerta al Ministerio de Salud para que se tomen las medidas necesarias y se identifiquen adecuadamente los factores que pudieran estar involucrados en este cuadro de gastroenteritis.

Las medidas de prevención que van desde un lavado de manos, adecuado desecho de excretas, potablidad del agua y la buena manipulación de alimentos son fundamentales para la disminución de casos de enteritis por Shigella spp.

En base a lo detallado anteriormente se presenta un análisis descriptivo de la información clínico epidemiológica y de laboratorio de los casos confirmados en el Laboratorio Clínico del Hospital de Los Chiles durante el período comprendido entre enero y diciembre del 2016.

\section{Materiales y métodos}

\section{Material biológico}

Se incluyeron 39 aislamientos de Shigella spp. provenientes de coprocultivos procesados en el Laboratorio Clínico del Hospital de Los Chiles durante todo el año 2016. Los microorganismos fueron aislados mediante procedimientos microbiológicos estandarizados e identificados por el equipo automatizado Vitek $2^{\circledR}$ de la casa francesa Biomeriux ${ }^{\circledR}$.

Seroagrupación: las bacterias aisladas compatibles con Shigella spp. fueron reaisladas en Agar Sangre e incubadas en aerobiosis por $18-24 \mathrm{hrs}$ a $37^{\circ} \mathrm{C}$ para la identificación del serogrupo. Se utilizaron antisueros polivalentes de dos grupos, S. sonnei y S. flexneri debido a que son las dos bacterias más frecuentes en nuestro país. El procedimiento se realizó de acuerdo a las indicaciones del fabricante.

Serotipificación y confirmación diagnóstica: los procedimientos de serotipificación y confirmación diagnóstica se realizaron en el Centro Nacional de Referencia de Bacteriología del Instituto Costarricense de Investigación y Enseñanza en Nutrición y Salud (INCIENSA). Para la serotipificación se utilizaron anticuerpos monoclonales.

Susceptibilidad a antimicrobianos: se realiza mediante el equipo automatizado Vitek 2 de la casa francesa Biomeriux.

\section{Resultados}

En la tabla 1 se muestran los aislamientos de Shigella spp. El 75\% de los aislamientos corresponden a Shigella sonnei, mientras que el $25 \%$ restante corresponden a Shigella flexneri. Es importante recalcar que no hubo aislamientos de $S$. dysenteriae ni S. boydii.

TABLA 1

Distribución de Shigella spp. aisladas en el Laboratorio Clínico del Hospital de los Chiles en el año 2016 según serogrupo

\begin{tabular}{lcc}
\multicolumn{1}{c}{ Serogrupo } & Número de aislamientos & Porcentaje \\
Shigella sonnei & 29 & 74.35 \\
Shigella flexneri & 10 & 25.65 \\
Shigella boydii & 0 & 0 \\
Shigella dysenteriae & 0 & 0 \\
\hline
\end{tabular}

Fuente: Laboratorio Clínico del Hospital de Los Chiles, 2017. 
TABLA 2

Resistencia antimicrobiana de Shigella flexneri aisladas en el Laboratorio Clínico del Hospital de Los Chiles durante el año 2016

\begin{tabular}{lccc}
\multicolumn{1}{c}{ Antibiótico } & Test realizados & Total resistentes & Porcentaje resistentes \\
Ácido Nalidíxico & 10 & 0 & $0 \%$ \\
Ampicilina & 10 & 9 & $90.0 \%$ \\
Cefotaxime & 10 & 0 & $0 \%$ \\
Ceftazidima & 10 & 0 & $0 \%$ \\
Ciprofloxacina & 10 & 0 & $0 \%$ \\
Gentamicina & 10 & 10 & $100 \%$ \\
Trimetoprim/Sulfametoxazole & 10 & 5 & $50.0 \%$ \\
\hline
\end{tabular}

Fuente: Laboratorio Clínico del Hospital de Los Chiles, 2017.

Los resultados de las pruebas de sensibilidad de $S$. flexneri se muestran en la tabla 2 . Se puede observar que el $100 \%$ de las cepas aisladas son resistentes a Gentamicina, mientras que muestra una resistencia del $90 \%$ y $50 \%$ a Ampicilina y al Trimetropin/Sulfametoxazole respectivamente. Ninguno de los aislamientos presentó betalactamasas de espectro extendido (BLEE) y presentaron un $100 \%$ de sensibilidad para el resto de antibióticos utilizados. En el caso de $S$. sonnei tal y como se muestra en el tabla 3, el $100 \%$ de los aislamientos presentaron resistencia a Gentamicina, y a Trimetropin/ Sulfametoxazole, el $69 \%$ de los aislamientos fueron resistentes a Ampicilina, mientras que el $100 \%$ de los aislamientos fueron sensibles al resto de antibióticos utilizados.

Según la Norma CLSI 2012 para Shigella spp., los aminoglucósidos (gentamicina) pueden aparecer activos "in vivo", pero no son efectivos clínicamente por lo que se debe omitir su interpretación, así mismo, esta misma norma no indica puntos de corte para el Ácido Nalidíxico en cepas aisladas de orígenes distintos al tracto urinario, por lo que también se debe omitir su interpretación.

Según los datos recopilados del total de aislamientos de Shigella spp., el distrito de Los Chiles es el que muestra una mayor cantidad de aislamientos con 26 casos de los 39 reportados, mientras que en el Amparo se presentaron cuatro casos, cinco en San Jorge, dos en Caño Negro y dos casos de Pocosol, este último no pertenece al Cantón de Los Chiles.

Con respecto a los grupos etarios afectados por las infecciones de Shigella spp., se puede observar en la tabla 4 que el grupo más aquejado fue el pediátrico con un 51,3\% de los casos, seguido por el de adultos $(25,7 \%)$, adolescentes $(20,5 \%)$ y mayores de 60 años $(2,5 \%)$.

TABLA 3

Resistencia antimicrobiana de Shigella sonnei aisladas en el Laboratorio Clínico del Hospital de Los Chiles durante el año 2016

\begin{tabular}{lccc}
\multicolumn{1}{c}{ Antibiótico } & Test Realizados & Total resistentes & Porcentaje resistentes \\
Ácido Nalidíxico & 29 & 0 & $0.0 \%$ \\
Ampicilina & 29 & 20 & $69.0 \%$ \\
Cefotaxime & 29 & 0 & $0.0 \%$ \\
Ceftazidima & 29 & 0 & $0.0 \%$ \\
Ciprofloxacina & 29 & 0 & $0.0 \%$ \\
Gentamicina & 29 & 29 & $100.0 \%$ \\
Trimetoprim/Sulfametoxazole & 29 & 29 & $100.0 \%$ \\
\hline
\end{tabular}

Fuente: Laboratorio Clínico del Hospital de Los Chiles, 2017. 
TABLA 4

Cantidad de casos de Shigella spp. reportados según el grupo etario

\begin{tabular}{ccc} 
Grupo etario (años) & Cantidad de casos & Porcentaje (\%) \\
$0-12$ & 20 & 51.3 \\
$13-20$ & 8 & 20.5 \\
$21-60$ & 10 & 25.7 \\
$>60$ años & 1 & 2.5 \\
\hline
\end{tabular}

Fuente: Laboratorio Clínico del Hospital de Los Chiles, 2017.

\section{Discusión}

Según los datos recopilados de los aislamientos realizados durante todo el año 2016, se muestra un claro predominio de $S$. sonnei $(75 \%)$ con respecto a $S$. flexneri (25\%). En general $S$. flexneri es más frecuente en países en desarrollo y $S$. sonnei lo es en países desarrollados (Organización Mundial de la Salud, 2005, Von $\&$ Mohammad, 2006). No obstante, nuestro sistema de salud y el suministro de agua potable podrían ser la causa de que la epidemiología sea similar a la de un país desarrollado.

La identificación del serogrupo y serotipo en conjunto con la determinación del perfil de la susceptibilidad antimicrobiana juegan un rol fundamental para la toma de decisiones terapéuticas y la aplicación de medidas de control apropiadas (Baca et al., 2013). La mayoría de diarreas son autolimitadas requiriendo solamente una adecuada rehidratación oral, sin embargo, el uso de antibióticos ha sido generalizado, justificando su uso por el hecho de presencia de fiebre, exámenes de laboratorio alterados, presión de los padres para acortar el período de enfermedad, lo cual ha generado una creciente multiresistencia antimicrobiona de Shigella spp (Mejía, 2007).

Con relación a la susceptibilidad antimicrobiana, los resultados muestran una elevada resistencia a antibióticos de prescripción tradicional como el Trimetropin/Sulfametoxale, mostrando una ligera diferencia con el resto del país, ya que el INCIENSA reporta una resistencia a nivel nacional del $96 \%$ en S. sonnei y del $74 \%$ en $S$. flexneri, mientras que la resistencia a nivel local fue del $100 \%$ y $50 \%$ respectivamente. La Ceftazidime, una cefalosporina de tercera generación, presenta altos niveles de sensibilidad, sin embargo, su uso está restringido al tratamiento de infecciones de mayor gravedad a fin de evitar el incremento de la resistencia a esta generación de antibióticos (Organización Mundial de la Salud, 2005). Actualmente, el tratamiento recomendado para la shigelosis es la Ciprofloxacina (Bolaños et al., 2013, Mejía, 2007), antimicrobiano al que el $100 \%$ de los aislamientos fueron sensibles.

Según los datos recopilados, el grupo etáreo más afectado fue el de los niños menores de 12 años con más de la mitad de los casos confirmados durante todo el año 2016. De manera similar, el INCIENSA reportó un 52\% de casos confirmados a nivel nacional durante el primer trimestre del 2014 en pacientes menores de 15 años (Bolaños et al., 2014). Es importante recalcar que la gravedad de la infección está relacionada a factores como la edad, afectando principalmente a niños menores de 5 años (Mejías, 2007) con una mayor mortalidad en este grupo etáreo (Barrantes y Achí, 2009). Se ha estimado que anualmente ocurren 1 billón de episodios de diarrea en todo el mundo en niños menores de 5 años, ocasionando 5 millones de casos fatales (Ramírez, 2002).

Según los resultados del Censo Nacional de Población y Vivienda del 2011, el cantón de Los Chiles está en la posición 79 de 81 cantones en cuanto al Índice de Desarrollo Humano, además, presenta un $25,3 \%$ de pobreza $(21,3 \%$ Nacional) y $8,4 \%$ de pobreza extrema $(6.0 \%$ Nacional) (Instituto Nacional de Estadística y Censos, 2011).Este cantón, tiene una extensión de 1358,67 Km cuadrados y está divido en cuatro distritos que son: Los Chiles, El Amparo, 
Caño Negro y San Jorge. La mayoría de casos se reportaron en el distrito de Los Chiles con un total de 26 cuadros de diarrea confirmados por Shigella spp, lo cual es lo esperado ya que el Hospital se encuentra a un kilómetro del centro de este lugar y porque concentra la mayor población con un total de 13253 habitantes. Mientras que en El Amparo se presentaron cuatro casos, en San Jorge cinco y en Pocosol dos, la menor proporción de casos puede ser atribuida a la menor cantidad de población de esos distritos ya que entre los tres distritos suman alrededor de 10500 habitantes. Por otro lado, las distancias que tienen que recorrer para ser atendidos en el hospital van desde los 19 kilómetros hasta los 40 kilómetros de distancia, por lo que se podrían dejar de captar casos debido al trayecto.

\section{Conclusiones y recomendaciones}

Dadas las condiciones socioeconómicas del cantón de Los Chiles y al ser zona limítrofe se hace necesario reforzar las actividades de promoción de la salud y atención en los primeros niveles de salud. Algunas acciones concretas serían promover y concientizar a la población sobre un adecuado lavado de manos y manejo de excretas, las personas con diarrea no deberían preparar alimentos y evitar el consumo de alimentos preparados en la vía pública.

El tratamiento en los cuadros de diarrea es la rehidratración con suero con la finalidad de reponer líquidos y reestablecer el balance electrolítico. El tratamiento antibiótico se debe reservar para los casos graves o para pacientes con desnutrición o que tienen comprometida su inmunidad como los pacientes con SIDA, individuos con quimioterapia $u$ otro tratamiento que afecte el estado inmune. Actualmente el antimicrobiano de elección para la disentería bacilar es la Ciprofloxacina, sin embargo, el médico debe apoyarse en el antibiograma brindado por el laboratorio clínico.

La vigilancia epidemiológica junto con los reportes oportunos del laboratorio clínico son fundamentales para establecer medidas de prevención, contención y control ya que permiten identificar las formas de transmisión, zonas afectadas, determinar condiciones de vivienda y establecer un cerco epidemiológico que impida la diseminación de la bacteria.

\section{Referencias}

Baca, C., Yupanqui, L., Canales, J., Zamudio, L., Quispe, M. Y Tamariz, J. (2013). Serotipos y susceptibilidad antimicrobiana de Shigella aisladas en un instituto de salud pediátrico de Lima, Perú entre enero y julio 2013. Rev Med Hered, 25, 73-79.

Barrantes, K Y Achí, R. (2009). Interacciones celulares en el proceso de invasión de Shigella sp. Rev Panam Infectol, 11(2), 56-61.

Bernardes, M., Nogueira, E., Birchal, G., Peret-Filho, L., Penna, F. \& Prazeres, P. (2013). Shigella in Brazilian children with acute diarrhoea: prevalence, antimicrobial resistance and virulence genes. Rio de Janeiro: Department of Microbiology, Institute of Biological Sciences. Mem Inst Oswaldo Cruz, 108(1), 30-35.

Bolaños, H., Tijerino, A., Oropeza, G., Morales, J., Campos, E. y Red Nacional de Laboratorios de Bacteriología. (2014). Incremento significativo de la shigelosis en Costa Rica durante el primer cuatrimestre del 2014. INCIENSA.1-16.

Clinical and Laboratory Stadards Institute, CLSI. (2014). Performance Standards for Antimicrobial Susceptibility Testing. M100-S24 Vol 34.

Decreto Ejecutivo No. 37.306-S. Reglamento de Vigilancia de la Salud. Alcance digital No. 160 a la Gaceta del 22 de octubre de 2012.

Del piano, L., Tejerina, H., Conat, E. y Aviles, C. (2001). Patrones de sensibilidad in vitro y comportamiento clínico de Shigella. Rev Chil Infect, 18(2), 101-107.

Instituto Nacional de Estadística y Censos, INEC. (2011). $\mathrm{X}$ Censo Nacional de población y Vl de vivienda 2011. Resultados generales. INEC. 1-142.

Keddy, H., Sooka, A., Crowther-Gibson, P., Quan, V., Meiring, S. \& Cohen, C. (2012). Systemic shigellosis in South Africa. Clin Infect Dis; 54, 1448-1454.

Lampel, K. \& Maurelli, A. (2003). Shigella species. Ch 11 In: Miliotis MD, Bier JW (eds) International handbook of foodborne pathogens. Marcel Dekker, New York, 167-180. 
Mejía, H. (2007). Opciones de tratamiento en Shigelosis. Rev Soc Bol Ped, 46(1), 80-84.

Mota, M., Gadea, M., Gonzalez, S., Gonzalez, G., Pardo, L. \& Sirok, A. (2010). Bacterial pathogens associated with bloody diarrhea in Uruguayan children. Rev Argent Microbiol; 42, 114-117.

Prats, G. Y Mirelis, B. (n.d). Género Shigella: aspectos prácticos para el laboratorio de microbiología. Control Calidad SEIMC. Hospital de Sant Pau, Barcelona.

Public Health England. (2015). UK standards for microbiology investigations, identification of Shigella species. Bacteriology-identification, Issue no: 3.
Ramírez, S. (2002). Shigelosis (disentería bacilar). Salud en tabasco, 8(1), 22-25.

Von, R. \& Mohammad, A. (2006). A multicentre study of Shigella diarrea in six Asian countries: disease burden, clinical manifestations, and microbiology. PLoS Medicine, 3, 1556-1569.

World Health Organization. (2005) Guidelines for the control of shigellosis, including epidemics due to Shigella dysenteriae type 1. Geneve, Switzerland: World Healt Organization Library Cataloguing-inPublication-Data. 